\title{
Article
}

\section{Pre- and Perinatal Characteristics Associated with Apgar Scores in a Review and in a New Study of Dutch Twins}

\author{
Veronika V. Odintsova ${ }^{1,2,3, \star}$, Conor V. Dolan ${ }^{1}$, Catharina E. M. van Beijsterveldt ${ }^{1}$, Eveline L. de Zeeuw ${ }^{1}$, Jenny van Dongen ${ }^{1}$ \\ and Dorret I. Boomsma ${ }^{1}$ \\ ${ }^{1}$ Department of Biological Psychology, Vrije Universiteit Amsterdam, Amsterdam, the Netherlands, ${ }^{2}$ Directorate, VI Kulakov National Medical Research Center for \\ Obstetrics, Gynecology and Perinatology, Moscow, Russia and ${ }^{3}$ Mother and Child Health Care Department, Federal Research Institute for Health Organization \\ and Informatics, Moscow, Russia
}

\begin{abstract}
A literature review was carried out to identify pre and perinatal characteristics associated with variation in Apgar scores in population-based studies. The parameters identified in the literature search were included in the classical twin design study to estimate effects of pre and perinatal factors shared and nonshared by twins and to test for a contribution of genetic factors in 1- and 5-min Apgar scores in a large sample of Dutch monozygotic (MZ) and dizygotic (DZ) twins. The sample included MZ and DZ twins $(N=5181$ pairs) recruited by the Netherlands Twin Register shortly after birth, with data on prenatal characteristics and Apgar scores at first and/or fifth minutes. The ordinal regression and structural equation modeling were used to analyze the effects of characteristics identified in the literature review and to estimate genetic and nongenetic variance components. The literature review identified 63 papers. Consistent with the review, we observed statistically significant effects of birth order, zygosity and gestational age (GA) for 1- and 5-min Apgar scores of both twins. Apgar scores are higher in first-born versus second-born twins and DZ first-born versus MZ first-born twins. Birth weight had an effect on the 5-min Apgar of the first born. Fetal presentation and mode of delivery had different effects on Apgar scores of first- and second-born twins. Parental characteristics and chorionicity did not have significant main effects on Apgar scores. The MZ twins' Apgar correlations equaled the DZ Apgar correlations. Our analyses suggest that individual differences in 1- and 5-min Apgar scores are attributable to shared and nonshared pre and perinatal factors, but not to genotypic factors of the newborns. The main predictors of Apgar scores are birth order, zygosity, GA, birth weight, mode of delivery and fetal presentation.
\end{abstract}

Keywords: Twin study; Apgar score; twin delivery; prenatal; perinatal; heritability; chorionicity

(Received 12 April 2019; accepted 18 April 2019)

The Apgar score is an important indicator of a newborn's health and is established immediately after birth (Apgar, 1953). Apgar scoring is standard in obstetrics and neonatological practice, and the advantage of this screening tool is that it allows for prompt standardized assessment (Apgar, 1953; Committee on Obstetric Practice American Academy of Pediatrics - Committee on Fetus and Newborn, 2015). It has proven its utility as a population-level indicator of outcome risk, with predictive value for neonatal and infant death and post neonatal development (Apgar, 1966; Drage et al., 1964; Harrington et al., 2007; Nelson \& Ellenberg, 1981). Its value has been confirmed in national register studies (Casey et al., 2001; Grunebaum et al., 2013; Iliodromiti et al., 2014; Li et al., 2013; Straube et al., 2010; ThorngrenJerneck \& Herbst, 2001) and is recognized by national guidelines and the World Health Organization (Siddiqui et al., 2017; WHO, 2012). However, there is a discussion regarding other, possibly

Author for correspondence: Veronika V. Odintsova, Email: v.v.odintsova@vu.nl Cite this article: Odintsova VV, Dolan CV, van Beijsterveldt CEM, de Zeeuw EL, van Dongen J, and Boomsma DI. (2019) Pre- and Perinatal Characteristics Associated with Apgar Scores in a Review and in a New Study of Dutch Twins. Twin Research and Human Genetics 22: 164-176, https://doi.org/10.1017/thg.2019.24 more precise, monitoring tools (O’Donnell et al., 2006; Rudiger et al., 2015). Apgar scores are evaluated at the first and fifth minutes after birth. While a low first-minute score is often transitory, persistence of poor health, resulting in a low fifth-minute score, often implies complications of clinical importance and indicates that the newborn has not responded to initial intervention (Drage et al., 1964; Harrington et al., 2007; Kattwinkel et al., 2010; Siddiqui et al., 2017; Thorngren-Jerneck \& Herbst, 2001).

The assessment of Apgar scores in twins was first presented by Virginia Apgar in 1953. For twins, the Apgar score of the first-born twin typically was better than that of the second-born twin (Apgar, 1953). Replication studies of differences among first- and secondborn twins (Haest et al., 2005; Herbst \& Kallen, 2008; Morley et al., 1989; Wen et al., 2004), among twins and singletons (Hegyi et al., 1998; Thorngren-Jerneck \& Herbst, 2001), among term and preterm newborns (Dolgun et al., 2016; Morley et al., 1989) and among different presentation and modes of delivery (Rossi et al., 2011) followed this initial work. Two small studies (Franchi-Pinto et al., 1999; Riese, 1990) suggested that genetic factors also contribute to variation in Apgar scores. However, because of small sample sizes, the results of these studies were inconclusive. 
The aim of the present study is twofold. First, we present a literature review of studies of Apgar scores, including singleton and multiple births, to identify pre and perinatal characteristics associated with variation in Apgar scores in population-based studies. Second, in a large sample of Dutch monozygotic (MZ) and dizygotic (DZ) twins enrolled in the Netherlands Twin Register (NTR) (van Beijsterveldt et al., 2013), we estimated effects of pre and perinatal factors shared and nonshared by twins, including the parameters identified in the literature search and test for a contribution of genetic factors in the classical twin design.

\section{Methods}

\section{Literature Review}

A review of the literature regarding pre and perinatal characteristics associated with Apgar scores was conducted in PubMed (MEDLINE), Web of Science, Embase and reference lists of retrieved articles. Search terms were 'Apgar scores' and 'heritability,' 'genetic effect,' 'prenatal factors,' 'twins,' 'fetal presentation, ' mode of delivery,' 'gestational age' and 'neonatal outcome'. Studies on specific clinical aspects of pregnancy and neonatology and on mortality and morbidity were excluded. We followed prior research on Apgar scores (Milsom et al., 2002; Sibony et al., 2006; Straube et al., 2010) and grouped pre and perinatal characteristics in the following categories - biological maternal and paternal factors, socioeconomic factors, mode of conception, gestational age (GA), pregnancy and delivery characteristics and newborn characteristics. The list of characteristics was included in an empirical study in which resemblance in MZ and DZ twins in a bivariate (1- and 5-min Apgar scores) model was evaluated.

\section{Empirical Study in Twins: Data Collection}

Data on Apgar scores and pre and perinatal characteristics were obtained from the NTR (van Beijsterveldt et al., 2013). The NTR recruits families with twins a few weeks to months after birth. Informed consent is obtained from parents. Surveys, including questions on pregnancy, birth and outcomes, were sent to mothers after registration of newborn twins.

\section{Zygosity}

For the majority of twin-pairs, genotyping for zygosity was based on a genome-wide single nucleotide polymorphism (SNP) array (Odintsova et al., 2018), or on genome-wide sets of microsatellites. Zygosity typing in earlier studies was based on smaller numbers of microsatellite markers, blood groups (van Dijk et al., 1996) or SNPs (van Beijsterveldt et al., 2013). For 27\% of the same-sex pairs, zygosity was based on items about physical similarity and frequency of confusion of the twins by parents and strangers from later surveys that correctly determine zygosity in $93 \%$ of the cases (Rietveld et al., 2000). In 19\% of the cases, zygosity was based on a single item, indicating how much the children look alike at age 2 , which gives a correct determination of zygosity in $92 \%$ of the cases (Groen-Blokhuis et al., 2011). For the other same-sex pairs, zygosity was based on a single question from survey 1 .

The sample comprised 5181 twin pairs born between 2005 and 2017. Of these, 1763 were MZ and 3418 were DZ (34\% and 66\%, respectively, reflecting population prevalence in the Netherlands). The data set includes complete information on zygosity, GA and time between birth of the first and second twins. One-min Apgar scores were available in 4947 pairs and 5-min Apgar were available scores in 4724 pairs. Both Apgar scores were available for 4623 pairs.
The study protocols were approved on March 16, 2004, by the Central Ethics Committee on Research Involving Human Subjects of the VU University Medical Center, Amsterdam; and May 25, 2017 (NTR-25-mei-2007). All participants provided informed consent.

\section{Variables}

Apgar scores were analyzed: (a) as a continuous variable (scores between 0 and 10); (b) as conventional categories (ordinal variables): Apgar values of 0-6 (low), 7-9 (intermediate) and 10 (high); a total score of lower than 7 is considered a source of concern (Committee on Obstetric Practice American Academy of Pediatrics - Committee on Fetus and Newborn, 2015).

Based on the literature review, we tested for the effects of birth order (first or second born), zygosity (MZ or DZ), sex (boys or girls), GA, birth weight, mother's and father's age at birth, mother's body mass index (BMI) at birth, fetal presentation (head presentation: cephalic; breech and horizontal presentations: noncephalic), mode of delivery (vaginal and intervention with vacuum extraction, forceps or cesarean section), and intertwin delivery interval. For $1003 \mathrm{MZ}$ twins, we had information on chorionicity (van Beijsterveldt et al., 2016). Of these, 745 were monochorionic (MC) and 258 were dichorionic (DC).

\section{Data Analyses}

Frequencies and means. The data were analyzed using SPSS version 25. The frequencies of maternal, delivery and infant characteristics were obtained within each Apgar score category, for first- and second-born twins and for MZ and DZ pairs. Differences between continuous variables in $\mathrm{MZ}$ and $\mathrm{DZ}$ pairs were tested using ANOVA, comparisons between first and second born with paired $t$ tests.

Fixed effect analysis. The role of maternal, pregnancy, delivery and infant characteristics were analyzed in the first- and second-born twin by ordinal regression. The significant characteristics were included in genetic covariance structure (GCS) analysis of the twin data. Four variables (GA, fetal presentation, mode of delivery and birth weight) were selected for inclusion in the analyses of twin resemblance for 1- and 5-min Apgar scores.

Twin correlations. Twin (polychoric) correlations of ordinal Apgar scores in $\mathrm{MZ}$ and $\mathrm{DZ}$ twins were estimated in Mplus. MZ twins are genetically identical, while DZ twins share on average $50 \%$ of their alleles identical by descent (from their parents). The MZ correlation (rMZ) is expected to be greater than the $\mathrm{DZ}$ correlations ( $\mathrm{rDZ}$ ) if the phenotype is influenced by genes ( $\mathrm{rMZ}>\mathrm{rDZ}$ ). The presence of shared environmental factors is suggested if the $\mathrm{DZ}$ correlation is larger than half the $\mathrm{MZ}$ correlation $(\mathrm{rDZ}>\mathrm{rMZ} / 2)$. Unshared environmental influences are present if the MZ correlation is less than 1 (Boomsma et al., 2002).

GCS modeling. We carried out GCS analyses of polychoric correlation matrices of the ordinal (3-point) 1- and 5-min Apgar scores using Mplus 6 (Muthen \& Muthen, 2007). The analysis of ordinal data is based on the liability-threshold model (Falconer, 1993), in which the ordinal scores arise by imposing thresholds on a continuous (standard normal) liability dimension. The twin resemblance at the level of this dimension is expressed by the polychoric correlations. The thresholds are a function of frequencies of the ordinal Apgar values. Given the 3-point ordinal Apgar scores, 
as there are two thresholds in GCS analysis, we first fitted models to estimate the polychoric correlation matrices in the MZ and DZ twins and to analyse the thresholds in the presence of the covariates. Subsequently, we fitted an ACE model (see below), in which the phenotypic polychoric correlations are model in terms of genetic and shared and unshared environmental effects. Parameter estimates were obtained by means of the weighted least squares estimation (the Mplus estimator WLSMV; Muthen \& Muthen, 2007). Model comparisons were based on the comparison of model chi-square (goodness of fit) statistics, using the Mplus difference test procedure. The main aim of the GCS modeling was to assess the contributions of genetic and environmental influences to the phenotypic (co)variance matrix of the first- and fifth-minute Apgar scores, while correcting for relevant covariates. We fitted a bivariate ACE model, which included additive genetic (A), shared environmental (C) and unshared environmental effects (E). The results of the analyses provide us with the decomposition of the phenotypic variance of the 1- and 5-min Apgar scores and the decomposition of the phenotype covariance (1-min with 5-min Apgar scores) into genetic and environmental components. In fitting the bivariate ACE model, we used a chi-square difference test to test sex differences, zygosity difference and birth-order differences in the covariates. We also tested sex differences in the ACE variance components.

We first fitted a model in which the thresholds differed with respect to birth order (first- vs. second-born twin), sex and zygosity (see Table S2 in the Supplementary material). We tested whether the thresholds were equal in males and females (retaining birth order and zygosity-related differences), which was found to be the case, $\chi^{2}(16)=23.8, p=.09$. We tested whether the thresholds were equal in $\mathrm{MZ}$ and $\mathrm{DZ}$ twins, but this was not the case, $\chi^{2}(8)=23.4, p=.003$. So, in the model of choice, we estimated 16 thresholds: 2 for 1-min Apgar scores and 2 for 5-min Apgar scores, which were different for zygosity and birth order. In the model, the regression coefficients of the covariates differed with respect to birth order.

We fitted the bivariate ACE model next. In this model, we allowed for sex differences in the ACE covariance matrices. As the twin correlations are suggestive of a CE model (i.e., absence of additive genetic effects), we first tested whether we could fix the additive genetic parameters to zero. This was found to be the case: $\chi^{2}(6)=10.3, p=.11$. In this CE model, we constrained the shared and nonshared parameters to be equal over sex and found sex differences in the shared and nonshared parameters to be absent, $\chi^{2}(4)=3.958, p=.41$.

\section{Results}

\section{Review of the Literature}

The review of studies on characteristics associated with Apgar score included population-based and twin studies published from 1981 to 2018, with exclusion of studies that concern specific clinical groups (e.g., preeclampsia, gestational diabetes), mortality and morbidity in newborns, and long-term outcomes associated with Apgar scores. Our literature search identified 63 studies, including studies of twins (see Table 1) and singletons (see Table S1 in the Supplementary material). The characteristics associated with Apgar scores may be summarized as:

Biological maternal and paternal factors. Maternal short stature (Camilleri, 1981; Svenvik et al., 2015), low maternal age $<17$ (X. K. Chen et al., 2007), high maternal age $>40$ years (Jahromi
\& Husseini, 2008; Straube et al., 2010) and high paternal age $>55$ years (Khandwala et al., 2018) were associated with low Apgar scores. However, Milsom et al. (2002) found no association of Apgar scores with maternal age. Higher mother's BMI and maternal obesity were associated with low Apgar scores in some (Chen et al., 2010; Straube et al., 2010; Thorngren-Jerneck \& Herbst, 2001), but not all studies (Kiran et al., 2005; Rode et al., 2005).

Socioeconomic factors. These were studied in European countries. Maternal occupation and single-parent status did not show an association with Apgar scores in the study of Straube et al. (2010). No significant association was found with employment status during pregnancy (Marbury et al., 1984; Milsom et al., 2002; Straube et al., 2010). Low Apgar scores were associated with single motherhood (Milsom et al., 2002), missing paternal demographic information (Tan et al., 2004), low level of mother's education and manual work (Hemminki et al., 1990; Odd et al., 2008) and adverse social circumstances (Kalland et al., 2006).

Mode of conception. Two small studies reported an association of 5-min Apgar score with mode of conception (Daniel et al., 2000; Ramoglu et al., 2014). No significant difference was found in terms of the 1- and 5-min Apgar scores between twins who were conceived naturally and twins who were conceived with the aid of artificial reproductive technologies (Caserta et al., 2014; Fan et al., 2013; Koudstaal et al., 2000; Moise et al., 1998; Ochsenkuhn et al., 2003; Pourali et al., 2016).

Gestational age. Apgar scores were associated with both low and high GA. For results on low GA, see Caukwell and Murphy (2002), Dolgun et al. (2016), Erdemoglu et al. (2003), Hartley \& Hitti (2005), Hegyi et al. (1998), Iliodromiti et al. (2014), Lindroos et al. (2018), Morley et al. (1989), Svenvik et al. (2015) and van der Ven et al (2014). For results on high GA, see Svenvik et al. (2015) and Thorngren-Jerneck \& Herbst (2001).

Pregnancy characteristics. A multiple pregnancy is a risk factor for adverse outcomes, including low Apgar scores (Morley et al., 1989; Pourali et al., 2016; Svenvik et al., 2015; ThorngrenJerneck \& Herbst, 2001). This holds specifically for MC pregnancies (Hjorto et al., 2014; Lindroos et al., 2018) and DC pregnancies with discordant fetal weight (Vulic et al., 2017). Again, some studies failed to find an association with chorionicity (Machado et al., 2017). Even though maternal smoking is generally associated with negative outcomes in neonates, most studies found no significant association between Apgar scores and prenatal maternal smoking after accounting for other confounders (Gilman et al., 2008; Kalland et al., 2006; Milsom et al., 2002; Straube et al., 2010), although an association between mother's smoking during the first trimester and low Apgar score was seen by Kallen (2001).

Delivery characteristics. Multiple deliveries were associated with adverse outcomes in the second-born twin (Haest et al., 2005; Herbst \& Kallen, 2008; Kwon et al., 2011; Morley et al., 1989; Thorngren-Jerneck \& Herbst, 2001; Usta et al., 2002; Wen et al., 2004). The intertwin delivery interval is an important determinant of the adverse effects on the second born (Erdemoglu et al., 2003; Hartley \& Hitti, 2005; Hjorto et al., 2014; Kwon et al., 2011; Stein et al., 2008) as this interval is related to the risk of hypoxia, due to decreasing $\mathrm{pH}$ in the umbilical arterial blood. However, other studies showed that even a relatively long intertwin delivery interval was not associated with unfavorable Apgar scores (Algeri et al., 
Table 1. Review of studies on prenatal characteristics and Apgar scores in twins

\begin{tabular}{|c|c|c|c|c|c|c|c|}
\hline No, & Authors & Sample (country) & $\begin{array}{l}\text { Sample size } n \\
\text { infants ( } n \text { twin pairs) }\end{array}$ & Sample and setting & $\begin{array}{l}\text { Apgar score } \\
\text { (min) }\end{array}$ & $\begin{array}{l}\text { Characteristics associated with low Apgar } \\
\text { score }(\downarrow)\end{array}$ & $\begin{array}{l}\text { Characteristics not associated with Apgar } \\
\text { score }\end{array}$ \\
\hline 1 & $\begin{array}{l}\text { Rayburn } \\
\text { et al. (1984) }\end{array}$ & USA & 230 (115 pairs) & $\begin{array}{l}\text { Twin sample with vaginal } \\
\text { delivery }\end{array}$ & $5 \min$ & & Intertwin delivery time \\
\hline 2 & $\begin{array}{l}\text { Morley et al. } \\
\text { (1989) }\end{array}$ & UK & $\begin{array}{l}476 \text { (including } 45 \\
\text { twin pairs) }\end{array}$ & $\begin{array}{l}\text { Preterm sample singletons } \\
\text { and twins }\end{array}$ & $5 \min$ & Early GA, birth order (second twin) & Multiple birth in preterm \\
\hline 3 & $\begin{array}{l}\text { Hegyi et al. } \\
\text { (1998) }\end{array}$ & USA & $\begin{array}{l}1105 \text { (including } 123 \\
\text { twin pairs) }\end{array}$ & $\begin{array}{l}\text { Population-based cohort } \\
\text { of preterm births } \\
\text { (including multiplies) }\end{array}$ & $\begin{array}{l}1 \text { and } 5 \text { min } \\
\text { (components) }\end{array}$ & $\begin{array}{l}\text { Apgar } 1 \text { and } 5 \text { min: low birth weight, early } \\
\text { GA, lower arterial blood pH, race (black } \downarrow \text { vs. } \\
\text { white). Apgar } 1 \text { min: vaginal mode of } \\
\text { delivery, sex (male } \downarrow \text { vs. female) }\end{array}$ & \\
\hline 4 & $\begin{array}{l}\text { Moise et al. } \\
\text { (1998) }\end{array}$ & Israel & 120 (60 pairs) & $\begin{array}{l}\text { Case-control: twins after } \\
\text { IVF and spontaneous } \\
\text { conception }\end{array}$ & 1 and $5 \mathrm{~min}$ & & Mode of conception \\
\hline 5 & $\begin{array}{l}\text { Daniel et al. } \\
(2000)\end{array}$ & Israel & 594 (297 pairs) & $\begin{array}{l}\text { Twin pregnancies } \\
\text { conceived spontaneously } \\
\text { and via ART }\end{array}$ & 1 and $5 \mathrm{~min}$ & $\begin{array}{l}\text { Birth order with mode of conception ( } \downarrow \text { Apgar } \\
5 \text { min for the first twin ART-conceived) }\end{array}$ & \\
\hline 6 & $\begin{array}{l}\text { Koudstaal } \\
\text { et al. }(2000)\end{array}$ & The Netherlands & 288 (144 pairs) & $\begin{array}{l}\text { Case-control study: twins } \\
\text { after IVF and spontaneous } \\
\text { conception }\end{array}$ & $5 \min$ & & Mode of conception \\
\hline 7 & $\begin{array}{l}\text { Thorngren- } \\
\text { Jerneck \& } \\
\text { Herbst, } \\
\text { (2001) }\end{array}$ & Sweden & $\begin{array}{l}1,028,705 \text { (including } \\
6433 \text { twin pairs) }\end{array}$ & $\begin{array}{l}\text { Population-based cohort, } \\
\text { term infants including } \\
\text { multiplies }\end{array}$ & $5 \min$ & $\begin{array}{l}\text { Vaginal breech delivery, birth weights above } \\
5 \mathrm{~kg} \text {, second-born twins, primiparity, } \\
\text { maternal age, smoking, post date } \\
\text { pregnancy, epidural analgesia, male infant } \\
\text { gender, being born at night }\end{array}$ & \\
\hline 8 & $\begin{array}{l}\text { Caukwell \& } \\
\text { Murphy } \\
\text { (2002) }\end{array}$ & UK & 844 (422 pairs) & $\begin{array}{l}\text { Twins with different } \\
\text { presentation at birth }\end{array}$ & $5 \mathrm{~min}$ & Early GA & $\begin{array}{l}\text { Fetal presentation (cephalic/noncephalic) of } \\
\text { the second born with vaginal delivery }\end{array}$ \\
\hline 9 & $\begin{array}{l}\text { Usta et al. } \\
(2002)\end{array}$ & Lebanon & 922 (461 pairs) & Twin cohort, term & 1 and $5 \min$ & Birth order ( $\downarrow$ second born) & $\begin{array}{l}\text { in second-born mode of delivery or } \\
\text { presentation }\end{array}$ \\
\hline 10 & $\begin{array}{l}\text { Erdemoglu } \\
\text { et al. (2003) }\end{array}$ & Turkey & 252 (126 pairs) & Normal twin births & $\begin{array}{l}1 \text { and } 5 \mathrm{~min} \\
\text { of the second } \\
\text { born }\end{array}$ & $\begin{array}{l}\text { For second twin: early GA, low birth weight } \\
\text { of the second twin }(<1900 \mathrm{~g}) \text {, intertwin } \\
\text { delivery interval }>15 \mathrm{~min} \text { for second twin in } \\
\text { breech presentation }\end{array}$ & Delivery route, fetal presentation \\
\hline 11 & $\begin{array}{l}\text { Ochsenkuhn } \\
\text { et al. (2003) }\end{array}$ & Germany & $\begin{array}{l}477 \text { (including } 78 \\
\text { twin pairs) }\end{array}$ & $\begin{array}{l}\text { Case-control: twins after } \\
\text { IVF and spontaneous } \\
\text { conception }\end{array}$ & $\begin{array}{l}1,5 \text { and } 10 \\
\min \end{array}$ & & Mode of conception \\
\hline 12 & $\begin{array}{l}\text { Tan et al. } \\
\text { (2004) }\end{array}$ & USA & $\begin{array}{l}304,466(152,233 \\
\text { pairs })\end{array}$ & $\begin{array}{l}\text { Population-based cohort } \\
\text { twins }\end{array}$ & $5 \mathrm{~min}$ & Paternal demographic information missing & \\
\hline 13 & $\begin{array}{l}\text { Wen et al. } \\
(2004)\end{array}$ & USA & $\begin{array}{l}128,219 \text { second } \\
\text { twins ( } 128,219 \text { pairs })\end{array}$ & Live born second twins & $5 \mathrm{~min}$ & $\begin{array}{l}\text { Birth order and mode of delivery ( } \downarrow \text { in } \\
\text { second twin delivered with cesarean section } \\
\text { after vaginal delivery of the first twin) }\end{array}$ & \\
\hline 14 & $\begin{array}{l}\text { Haest et al. } \\
(2005)\end{array}$ & The Netherlands & 328 (164 pairs) & $\begin{array}{l}\text { Term twin births with } \\
\text { different mode of delivery }\end{array}$ & $5 \mathrm{~min}$ & Birth order ( $\downarrow$ in second twin) & $\begin{array}{l}\text { Mode of delivery (vaginal vs. planned } \\
\text { cesarean) }\end{array}$ \\
\hline 15 & $\begin{array}{l}\text { Hartley and } \\
\text { Hitti (2005) }\end{array}$ & USA & 10,276 (5,138 pairs) & $\begin{array}{l}\text { Twin births with different } \\
\text { delivery interval (low risk } \\
\text { group) }\end{array}$ & $5 \mathrm{~min}$ & $\begin{array}{l}\text { Early GA; birth order ( } \downarrow \text { in second twin); for } \\
\text { second twin long intertwin delivery interval } \\
>15 \text { min, fetal breech presentation }\end{array}$ & \\
\hline
\end{tabular}




\begin{tabular}{|c|c|c|c|c|c|c|c|}
\hline No, & Authors & Sample (country) & $\begin{array}{l}\text { Sample size } n \\
\text { infants ( } n \text { twin pairs) }\end{array}$ & Sample and setting & $\begin{array}{l}\text { Apgar score } \\
\text { (min) }\end{array}$ & $\begin{array}{l}\text { Characteristics associated with low Apgar } \\
\text { score }(\downarrow)\end{array}$ & $\begin{array}{c}\text { Characteristics not associated with Apgar } \\
\text { score }\end{array}$ \\
\hline 16 & $\begin{array}{l}\text { Usta et al. } \\
\text { (2005) }\end{array}$ & Lebanon & 434 (217 pairs) & Twin cohort & 1 and5 $\min$ & $\begin{array}{l}\text { Vaginal delivery of vertex-nonvertex twins ( } \downarrow \\
\text { in second twin) }\end{array}$ & \\
\hline 17 & $\begin{array}{l}\text { Sibony et al. } \\
(2006)\end{array}$ & France & 1228 (614 pairs) & $\begin{array}{l}\text { Twin cohort preterm and } \\
\text { term }\end{array}$ & $5 \mathrm{~min}$ & $\begin{array}{l}\text { mode of delivery: cesarean section for } \\
\text { second born }\end{array}$ & \\
\hline 18 & $\begin{array}{l}\text { Bjelic- } \\
\text { Radisic et al. } \\
\text { (2007) }\end{array}$ & Austria & 562 (281 pairs) & Twin birth cohort & $\begin{array}{l}1,5 \text { and } 10 \\
\min \end{array}$ & $\begin{array}{l}\text { Birth order ( } \downarrow \text { in second twin), for second } \\
\text { twin mode of delivery ( } \downarrow \text { cesarean section } \\
\text { after vaginal delivery of first born or vaginal } \\
\text { after vaginal of first born) }\end{array}$ & Fetal presentation of the second born \\
\hline 19 & $\begin{array}{l}\text { Sentilhes } \\
\text { et al. (2007) }\end{array}$ & France & 412 (206 pairs) & $\begin{array}{l}\text { Twin cohort with first twin } \\
\text { in breech position, term } \\
\text { deliveries }\end{array}$ & $5 \mathrm{~min}$ & & $\begin{array}{l}\text { Mode of delivery (vaginal vs. planned } \\
\text { cesarean) }\end{array}$ \\
\hline 20 & $\begin{array}{l}\text { Herbst \& } \\
\text { Kallen (2008) }\end{array}$ & Sweden & 31,982 (15,991 pairs) & $\begin{array}{l}\text { Uncomplicated twin } \\
\text { pregnancies with different } \\
\text { presentation and mode of } \\
\text { delivery }\end{array}$ & $5 \mathrm{~min}$ & $\begin{array}{l}\text { Birth order and mode of delivery ( } \downarrow \text { in } \\
\text { second born with vaginal delivery) }\end{array}$ & \\
\hline 21 & $\begin{array}{l}\text { Schmitz } \\
\text { et al. (2008) }\end{array}$ & France & 1516 (758 pairs) & $\begin{array}{l}\text { Twin cohort, term vaginal } \\
\text { deliveries, cephalic- } \\
\text { presenting first twin }\end{array}$ & $5 \mathrm{~min}$ & $\begin{array}{l}\text { Mode of delivery: planned vaginal in both } \\
\text { twins }\end{array}$ & Fetal presentation of the second born \\
\hline 22 & $\begin{array}{l}\text { Stein et al. } \\
(2008)\end{array}$ & Germany & 8220 (4110 pairs) & $\begin{array}{l}\text { Population-based twin } \\
\text { cohort, term vaginal } \\
\text { deliveries of first twin }\end{array}$ & $\begin{array}{l}1,5 \text { and } 10 \\
\min \end{array}$ & $\begin{array}{l}\text { Apgar score } 1,5 \text { and } 10 \text { min for second twin: } \\
\text { increased twin-to-twin delivery time interval. } \\
\text { Apgar score } 5 \text { min for second twin: birth } \\
\text { weight discordance (birth weight greater in } \\
\text { second twin), mode of delivery }\end{array}$ & \\
\hline 23 & $\begin{array}{l}\text { Fox et al. } \\
(2010)\end{array}$ & USA & 574 (287 pairs) & $\begin{array}{l}\text { Twin birth with different } \\
\text { mode of delivery }\end{array}$ & 1 and $5 \mathrm{~min}$ & Nonactive second-stage management & $\begin{array}{l}\text { Mode of delivery (vaginal vs. planned } \\
\text { cesarean) }\end{array}$ \\
\hline 24 & $\begin{array}{l}\text { Kwon et al. } \\
\text { (2011) }\end{array}$ & Korea & 158 (79 pairs) & $\begin{array}{l}\text { Twin births with known } \\
\text { umbilical arterial blood } \\
\text { parameters }\end{array}$ & 1 and $5 \mathrm{~min}$ & $\begin{array}{l}\text { Vaginal delivery and intertwin delivery time } \\
\text { ( } \downarrow \text { for second twin) }\end{array}$ & \\
\hline 25 & $\begin{array}{l}\text { Schneuber } \\
\text { et al. (2011) }\end{array}$ & Austria & 414(207 pairs) & Twin births, term deliveries & $\begin{array}{l}1,5 \text { and } 10 \\
\min \end{array}$ & & Intertwin delivery time \\
\hline 26 & $\begin{array}{l}\text { Barrett } \\
(2013)\end{array}$ & Canada & 5607 (2804 pairs) & Twin births & $5 \mathrm{~min}$ & & $\begin{array}{l}\text { Mode of delivery (planned vaginal vs. } \\
\text { planned cesarean section) on low Apgar as } \\
\text { the component of primary outcome }\end{array}$ \\
\hline 27 & $\begin{array}{l}\text { Fan et al. } \\
(2013)\end{array}$ & China & 750 (375 pairs) & $\begin{array}{l}\text { DC twin pregnancies } \\
\text { conceived spontaneously } \\
\text { and via ART }\end{array}$ & 1 and $5 \mathrm{~min}$ & & Mode of conception (spontaneous vs. ART) \\
\hline 28 & $\begin{array}{l}\text { Caserta } \\
\text { et al. (2014) }\end{array}$ & Italy & 690 (345 pairs) & $\begin{array}{l}\text { DC, diamniotic twin } \\
\text { pregnancies conceived } \\
\text { spontaneously and via ART }\end{array}$ & $5 \mathrm{~min}$ & & Mode of conception (spontaneous vs. ART) \\
\hline 29 & $\begin{array}{l}\text { Hjorto et al. } \\
\text { (2014) }\end{array}$ & Denmark & 1100 (550 pairs) & Twin deliveries & $1 \mathrm{~min}$ & $\begin{array}{l}\text { For second born chorionicity (MC), time } \\
\text { between birth, vacuum extraction }\end{array}$ & \\
\hline
\end{tabular}




\begin{tabular}{|c|c|c|c|c|c|c|c|}
\hline 30 & $\begin{array}{l}\text { Vogel et al. } \\
\text { (2014) }\end{array}$ & $\begin{array}{l}\text { WHO Global Survey on } \\
\text { Maternal Health } \\
\text { (multicountry Africa, Asia, } \\
\text { Latin America) }\end{array}$ & 2848 (1424 pairs) & Multiple births & $5 \mathrm{~min}$ & $\begin{array}{l}\text { For second twin: nonvertex presentation } \\
\text { after vaginal delivery of first twin }\end{array}$ & \\
\hline 31 & $\begin{array}{l}\text { Wenckus } \\
\text { et al. (2014) }\end{array}$ & USA & 4450 (2225 pairs) & Twin birth cohort & $5 \mathrm{~min}$ & $\begin{array}{l}\text { Mode of delivery (vaginal delivery } \downarrow \text { vs. } \\
\text { cesarian section) in twins }\end{array}$ & \\
\hline 32 & $\begin{array}{l}\text { Dolgun et al. } \\
\text { (2016) }\end{array}$ & Turkey & 176 (88 pairs) & $\begin{array}{l}\text { Preterm twins without } \\
\text { complications }\end{array}$ & 1 and $5 \mathrm{~min}$ & $\begin{array}{l}\text { Apgar score } 1 \text { min: low fetal body weight, } \\
\text { early GA, low height, head circumference }\end{array}$ & $\begin{array}{l}\text { Apgar } 1 \text { and } 5 \text { min: maternal age, placental } \\
\text { weight, length of umbilical cord, premature } \\
\text { rupture of membranes, birth order. Apgar } 5 \\
\text { min: gender, mode of delivery, GA, height, } \\
\text { head circumference. }\end{array}$ \\
\hline 33 & $\begin{array}{l}\text { Jhaveri \& } \\
\text { Nadkarni, } \\
\text { (2016) }\end{array}$ & India & 186 (93 pairs) & Twin birth sample & $5 \mathrm{~min}$ & For second-born vaginal mode of delivery & \\
\hline 34 & $\begin{array}{l}\text { Machado } \\
\text { et al. (2017) }\end{array}$ & Portugal & 1051 (540 pairs) & $\begin{array}{l}\text { Twin pregnancies with } \\
\text { known chorionicity }\end{array}$ & $5 \mathrm{~min}$ & & Chorionicity \\
\hline 35 & $\begin{array}{l}\text { Vulic et al. } \\
\text { (2017) }\end{array}$ & Croatia & 434 (217 pairs) & DC twin pregnancies & Not indicated & Discordant twin growth in DC pregnancies & \\
\hline 36 & $\begin{array}{l}\text { Pourali et al. } \\
\text { (2016) }\end{array}$ & Iran & 254 (127 pairs) & $\begin{array}{l}\text { DC twin pregnancies } \\
\text { following ART vs. } \\
\text { spontaneous }\end{array}$ & 1 and $5 \mathrm{~min}$ & Multiple pregnancy & Mode of conception (ART vs. spontaneous) \\
\hline 37 & $\begin{array}{l}\text { Zhao et al. } \\
\text { (2017) }\end{array}$ & USA & $\begin{array}{l}216,076(108,038 \\
\text { pairs })\end{array}$ & Mixed-gender twin pairs & $5 \mathrm{~min}$ & Male sex, mixed-gender twin pairs & \\
\hline 38 & $\begin{array}{l}\text { Algeri et al. } \\
\text { (2018) }\end{array}$ & Italy & 800 (400 pairs) & diamniotic pregnancies & Not indicated & & Intertwin delivery time \\
\hline 39 & $\begin{array}{l}\text { Lindroos } \\
\text { et al. (2018) }\end{array}$ & Sweden & 1054 (527 pairs) & $\begin{array}{l}\text { Twin birth with different } \\
\text { delivery interval (not high } \\
\text { risk group) }\end{array}$ & $5 \mathrm{~min}$ & $\begin{array}{l}\text { Lower birth weight, early GA, intertwin birth } \\
\text { weight discordance }>25 \% \text {, chorionicity (MC } \downarrow \\
\text { for second born) }\end{array}$ & $\begin{array}{l}\text { For second twin, twin-to-twin time interval } \\
\text { ( } 30 \mathrm{~min} \text { or }>30 \mathrm{~min} \text { ), presentation at birth }\end{array}$ \\
\hline
\end{tabular}



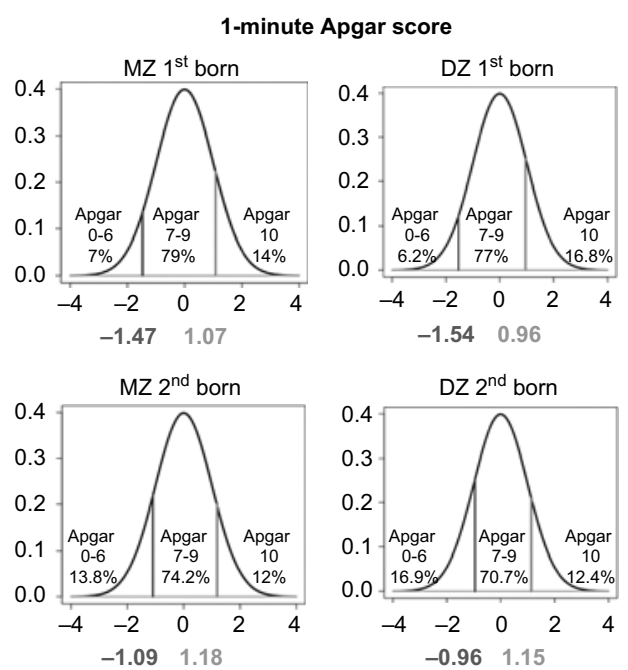
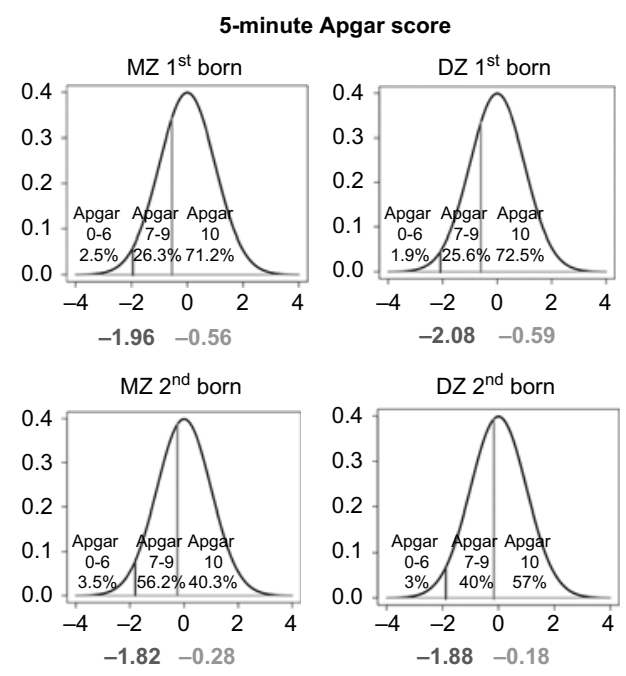

Fig. 1. Proportions of $M Z$ and $D Z$ newborn twins in different categories of 1- and 5-min Apgar score.
2018; Lindroos et al., 2018; Milsom et al., 2002; Rayburn et al., 1984; Schneuber et al., 2011).

Noncephalic (breech and horizontal) presentation at birth is associated with low Apgar score in singletons (Krebs \& Langhoff-Roos, 1999; Krebs et al., 2001; Vogel et al., 2014) and twins (Hartley \& Hitti, 2005). After a vaginal delivery of a vertex first twin, nonvertex presentation of the second twin was associated with increased odds of a low 5-min Apgar score (Vogel et al., 2014). However, other studies found no support for an effect of fetal presentation on the Apgar score of the second twin (BjelicRadisic et al., 2007; Caukwell \& Murphy, 2002; Lindroos et al., 2018; Schmitz et al., 2008; Usta et al., 2002). Emergency interventions (vacuum extraction, forceps and urgent operative delivery) were associated with adverse outcomes, including low Apgar scores (Hjorto et al., 2014; Milsom et al., 2002; Rode et al., 2005). The risk of a low Apgar score given at planned vaginal delivery was much higher than the risk associated with a selective cesarean section in singletons (Hegyi et al., 1998; Krebs \& Langhoff-Roos, 1999) and twins (Schmitz et al., 2008), especially in the second-born twin (Herbst \& Kallen, 2008; Jhaveri \& Nadkarni, 2016; Kwon et al., 2011; Usta et al., 2005; Wenckus et al., 2014). The effect of mode of delivery in twins was not supported by some studies analyzing different fetal presentation deliveries (Barrett, 2013; Fox et al., 2010; Sentilhes et al., 2007). Cesarean section was associated with low Apgar in the second twin following vaginal delivery of first twin (Sibony et al., 2006; Wen et al., 2004).

Newborn characteristics. Several studies found a positive association between birth weight and Apgar scores (Dolgun et al., 2016; Erdemoglu et al., 2003; Hegyi et al., 1998; Ladehoff et al., 1986; Lindroos et al., 2018; Stein et al., 2008; Thorngren-Jerneck \& Herbst, 2001). However, Iliodromiti et al. (2014) found no association of birth weight and Apgar scores in the large populationbased sample of more than 1 million births in Scotland. Birth weight discordance in twins is associated with low Apgar scores for the second born (Lindroos et al., 2018; Stein et al., 2008). On average, girls have higher Apgar scores than boys (Dolgun et al., 2016; Hegyi et al., 1998; Stevenson et al., 2000; Zhao et al., 2017).

Based on our literature review, we included the following risk factors in our analyses of the twin data: zygosity, chorionicity, birth order, GA, birth weight, sex, mother's age and father's age at birth, mother's BMI at birth, mode of delivery and fetal presentation.

\section{Descriptives and Apgar Scores in Twins}

Proportions of newborn NTR twins in three categories of 1- and 5- min Apgar scores (low, intermediate and high) for first- and second born in MZ and DZ twin pairs are presented in Figure 1 (distribution in pairs, see Table S3 in the Supplementary material). In terms of the proportions, the first- and second-born twins do not differ greatly with respect to the 1-min Apgar scores. For instance, the proportion of intermediate 1-min Apgar score (7-9) is about 79\%, $74 \%, 77 \%$ and $71 \%$ (MZ first born, $\mathrm{MZ}$ second born, DZ first born and DZ second born, respectively). There are, however, appreciable differences between the first- and second-born twins in the 5-min Apgar scores. For instance, the proportion of a high 1-min Apgar score (10) is about $71 \%, 40 \%, 72 \%$, and $57 \%$ (MZ first born, $\mathrm{MZ}$ second born, DZ first born and DZ second born, respectively).

We compared the means of Apgar scores in MZ and DZ twin pairs between first- and second-born twins. Mean continuous Apgar scores were higher in the first born than in second-born twins in both $\mathrm{MZ}$ and $\mathrm{DZ}$ pairs: in $\mathrm{MZ}$ at first minute, 8.45 versus $8.1(p<.0001)$, at fifth minute, 9.36 versus $9.22(p<.0001)$; in DZ at first minute, 8.61 versus $8.03(p<.0001)$, at fifth minute, 9.51 versus $9.26(p<.0001)$. MZ first-born twins had lower Apgar scores than DZ first-born twins: at first minute, 8.43 versus 8.6 in $\mathrm{DZ}$, at fifth minute, 9.36 versus 9.51 in $\mathrm{DZ}(p<.0001)$. In second-born twins there was no effect of zygosity (see Table S4 in the Supplementary material).

Next, we tested pre and perinatal characteristics for the firstand second-born twins. The distribution of perinatal and delivery characteristics of NTR twins are presented in Table 2 (for information on characteristics of low, intermediate and high Apgar score groups, see Table S5 in the Supplementary material). Given alpha of .05 , mother's age, father's age, and mother's BMI at birth did not predict Apgar scores (Table S6 in the Supplementary material). The intertwin delivery time was significant for Apgar scores of the second-born twin. There was no significant effect of chorionicity on Apgar score in MZ twins with chorionicity data, taking into account GA, birth weight, sex, mode of delivery and fetal presentation (Table S7 in the Supplementary material). Characteristics 
Table 2. Prenatal, delivery and infant characteristics of $M Z$ and $D Z$ twin Pairs

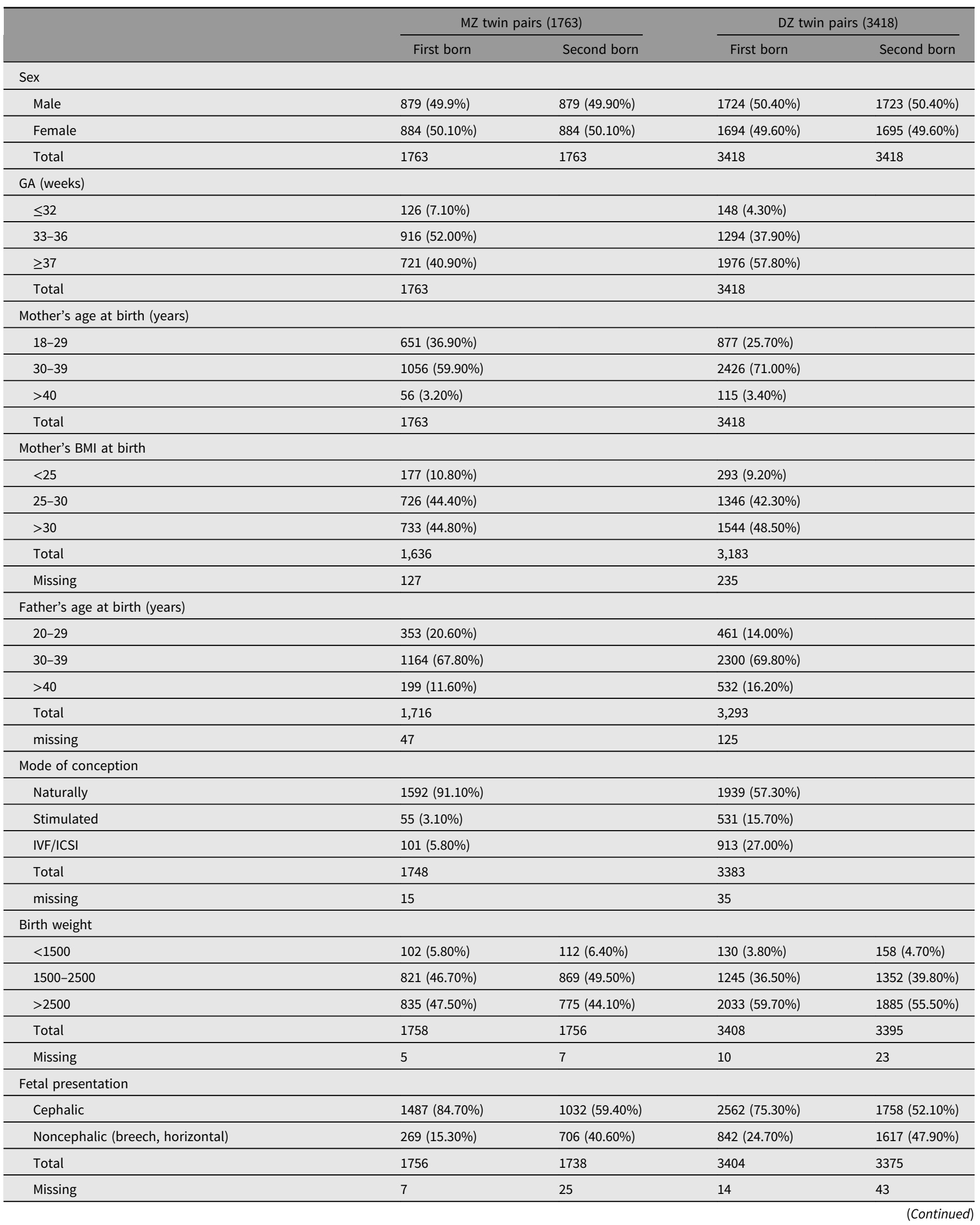


Table 2. (Continued)

\begin{tabular}{|c|c|c|c|c|}
\hline & \multicolumn{2}{|c|}{ MZ twin pairs (1763) } & \multicolumn{2}{|c|}{ DZ twin pairs (3418) } \\
\hline \multicolumn{5}{|l|}{ Mode of delivery } \\
\hline Planned cesarean section & $235(13.40 \%)$ & $234(13.40 \%)$ & $650(19.10 \%)$ & 649 (19.20\%) \\
\hline Urgent intervention (forceps, vacuum extraction) & $182(10.40 \%)$ & $110(6.30 \%)$ & $314(9.20 \%)$ & $257(7.60 \%)$ \\
\hline Total & 1,757 & 1,743 & 3410 & 3,386 \\
\hline Missing & 6 & 20 & 8 & 32 \\
\hline \multicolumn{5}{|l|}{ Intertwin delivery time (min) } \\
\hline$<5$ & $767(43.50 \%)$ & & $1492(43.70 \%)$ & \\
\hline $6-15$ & $612(34.70 \%)$ & & $914(26.70 \%)$ & \\
\hline
\end{tabular}

Note: Values are $n(\%)$.

without significant effects for both twins were excluded from further analysis.

Multigroup genetic covariance structural equation modeling of the ordinal 1- and 5-min Apgar scores included monozygotic male (MZM) and female (MZF), dizygotic male (DZM) and female (DZF), dizygotic male-female (DZMF) and dizygotic female-male (DZFM) pairs. We included GA (a characteristic of twin pairs) and fetal presentation, birth weight and mode of delivery (characteristics of individual twins) as covariates. We estimated the effects of covariates and the polychoric twin correlations (Table S2 in the Supplementary material).

In multigroup genetic analyses, the effects of covariates did not differ with respect to sex, $\chi^{2}(16)=8.88, p=.91$. GA had a positive effect on both Apgar measurements in both twins $(p<.0001$; Table 3). Birth weight had a positive effect on 5-min Apgar score of the first born $\left(\beta_{1 \mathrm{~min}}=.09, p=.002\right)$. The effects of delivery characteristics, such as mode of delivery and fetal presentation at birth, were different for first- and second-born twins. Noncephalic presentation at birth of the first-born twin had a positive effect on Apgar scores of the first born $\left(\beta_{1 \min }=.11, p=.02 ; \beta_{5 \min }=.19\right.$, $p<.0001)$ and noncephalic presentation of the second-born twin have negative effect on Apgar scores $\left(B_{1 \min }=-.23, p<.0001\right.$; $\left.\beta_{5 \min }=-.16, p<.0001\right)$. First-born twins delivered vaginally were more likely to have higher Apgar scores at both points $\left(B_{1 \mathrm{~min}}=.26\right.$, $\left.ß_{5 \min }=.42, p<.0001\right)$. Second-born twins delivered vaginally were more likely to have lower 1-min Apgar scores $\left(\beta_{1 \mathrm{~min}}=-.14\right.$, $p<.0001)$, and the effect was not significant for the 5-min Apgar score $\left(\beta_{5 \min }=-0.002, n s\right)$.

Table 4 summarizes the twin correlations with and without the correction for covariates (see also Table S8 in the Supplementary material). Overall, the correlations, which varied between .43 and .55 , did not differ greatly between zygosity, which suggests the absence of genetic effects. Shared environmental influences accounted for $52.6 \%$ (95\% confidence interval (CI) [0.51, 0.54]) and $50.2 \%$ (95\% CI $[0.48,0.52])$ of the variance of the $1-$ and $5-\mathrm{min}$ Apgar scores, respectively. The remainder of the variance was explained by nonshared environmental effects: $47.4 \%$ (95\% CI $[0.46,0.49])$ and $49.8 \%$ (95\% CI $[0.48,0.52])$ of the variance of the 1- and 5-min Apgar scores, respectively. The correlation between the 1- and 5-min Apgar scores was .70 (95\% CI [ 0.68, 0.72]), which is consistent with the correlations shown in Table 4 . This correlation is decomposed into .33 (95\% CI $[0.30,0.35])$ due to shared prenatal environmental factors, and .37 (95\% CI [0 .39, 0.40]) due to unshared prenatal environmental factors (see Table S9 and Figure S1 in the Supplementary material).

\section{Discussion}

Our literature review identified characteristics that were significantly associated with Apgar scores in population-based and twin studies. These included GA, birth weight, sex, mother's and father's age at birth, mother's BMI, mode of delivery, and fetal presentation, specifically for twins' zygosity, chorionicity, birth order and intertwin delivery time. In the current analyses of twins, birth order, zygosity, GA, birth weight, fetal presentation at birth and mode of delivery contributed to Apgar scores.

In our empirical study, parental characteristics were not associated with Apgar scores. The effects of mother's and father's age on Apgar scores as established in other studies may be explained by families included in these other studies, such as teenage mothers (X. K. Chen et al., 2007), mothers over age 40 (Jahromi \& Husseini, 2008), and older fathers (Khandwala et al., 2018). In our sample, only 171 women were above 40 years $(3.2 \% \mathrm{MZ}$ and $3.4 \% \mathrm{DZ}$ of mothers), and there were no mothers younger than 18 . An effect of a maternal BMI on Apgar score was found in some studies of singletons (M. Chen et al., 2010; Straube et al., 2010; ThorngrenJerneck \& Herbst, 2001). Multiple pregnancies are generally accompanied by greater maternal BMI than in singleton pregnancies due to gestational weight gain. This may explain the nonsignificance of maternal BMI effect on Apgar score in our study (near $45 \%$ of mothers in our sample had BMI $>30$ ). Optimal gestational weight gain in twin pregnancy is unclear (Bodnar et al., 2014). However, some studies also support our findings that maternal age (Milsom et al., 2002) and BMI at birth (Kiran et al., 2005; Rode et al., 2005) are not associated with Apgar score. Monochorionicity was an important risk factor for adverse perinatal outcomes in twins (Dube et al., 2002; Hjorto et al., 2014; Lindroos et al., 2018; 
Table 3. Average for continuous Apgar scores and effect of GA, birth weight, fetal presentation and mode of delivery on ordinal 1- and 5-min Apgar score in first- and second-born twins

\begin{tabular}{|c|c|c|}
\hline & $\beta$ SE Est./SE $p$ & $\mathrm{Cl}(95 \%)$ \\
\hline \multicolumn{3}{|l|}{ 1-min Apgar first-born twin: mean 8.45} \\
\hline \multicolumn{3}{|l|}{ Effect size for ordinal score } \\
\hline GA ( $z$ scores) & 0.2450 .0327 .4830 .000 & {$[0.161,0.329]$} \\
\hline Birth weight ( $z$ scores) & 0.0430 .0321 .4310 .153 & {$[-0.038,0.125]$} \\
\hline Fetal presentation ( $0=$ cephalic $/ 1=$ noncephalic) & 0.1100 .0482 .3220 .020 & {$[-0.014,0.233]$} \\
\hline Mode of delivery ( $0=$ vaginal $/ 1=$ intervention) & $-0.2580 .041-6.2970 .000$ & {$[-0.363,-0.152]$} \\
\hline \multicolumn{3}{|l|}{ 5-min Apgar first-born twin: mean 9.36} \\
\hline \multicolumn{3}{|l|}{ Effect size for ordinal score } \\
\hline GA ( $z$ scores) & 0.3010 .0339 .0000 .000 & {$[0.215,0.387]$} \\
\hline Birth weight ( $z$ scores) & 0.0970 .0323 .0550 .002 & {$[0.014,0.179]$} \\
\hline Fetal presentation (cephalic/noncephalic) & 0.1940 .0513 .8180 .000 & {$[0.062,0.325]$} \\
\hline Mode of delivery (vaginal/intervention) & $-0.4170 .041-10.2630 .000$ & {$[-0.522,-0.312]$} \\
\hline \multicolumn{3}{|l|}{ 1-min Apgar second-born twin: mean 8.61} \\
\hline \multicolumn{3}{|l|}{ Effect size for ordinal score } \\
\hline GA ( $z$ scores) & 0.2790 .0318 .9910 .000 & {$[0.200,0.360]$} \\
\hline Birth weight ( $z$ scores) & $-0.0010 .030-0.0120 .990$ & {$[-0.078,0.075]$} \\
\hline Fetal presentation (cephalic/noncephalic) & $-0.2310 .033-7.0420 .000$ & {$[-0.315,-0.147]$} \\
\hline Mode of delivery (vaginal/intervention) & 0.1410 .0344 .1000 .000 & {$[0.052,0.229]$} \\
\hline \multicolumn{3}{|l|}{ 5-min Apgar second-born twin: mean 9.51} \\
\hline \multicolumn{3}{|l|}{ Effect size for ordinal score } \\
\hline GA (z scores) & 0.3460 .03210 .9480 .000 & {$[0.265,0.428]$} \\
\hline Birth weight (z scores) & 0.0260 .0300 .8940 .372 & {$[-0.052,0.104]$} \\
\hline Fetal presentation (cephalic/noncephalic) & $-0.1630 .033-4.9660 .000$ & {$[-0.248,-0.079]$} \\
\hline Mode of delivery (vaginal/intervention) & 0.0020 .0340 .0700 .944 & {$[-0.086,0.091]$} \\
\hline
\end{tabular}

Note: $\mathrm{SE}=$ standard error, Est. $=$ estimates and $\mathrm{Cl}=$ confidence interval.

Table 4. Twin correlations for 1- and 5-min Apgar score (ordinal variables) noncorrected and corrected for GA, birth weight, mode of delivery and fetal presentation

\begin{tabular}{llll}
\hline & & $r$ & $r$ adjusted to covariates \\
\hline Twin correlations1-min Apgar & MZM & .621 & .552 \\
\hline & MZF & .607 & .537 \\
\hline DZM & .544 & .485 \\
\hline DZF & .610 & .551 \\
\hline DZMF & .539 & .479 \\
\hline DZFM & .595 & .536 \\
\hline MZM & .676 & .540 \\
\hline MZF & .643 & .507 \\
\hline DZM & .630 & .518 \\
\hline DZF & .620 & .508 \\
\hline DZMF & .568 & .455 \\
\hline DZFM & .547 & .435 \\
\hline
\end{tabular}

Note: $M Z M=$ monozygotic male, $M Z F=$ monozygotic female, $D Z M=$ dizygotic male, $\mathrm{DZF}=$ dizygotic female, DZMF = dizygotic male-female and DZFM = dizygotic female-male. van Beijsterveldt et al., 2016), but was not associated with Apgar scores in our study.

In accordance with many singleton (Hegyi et al., 1998; Iliodromiti et al., 2014; Svenvik et al., 2015; van der Ven et al., 2014) and twin studies (Caukwell \& Murphy, 2002; Dolgun et al., 2016; Erdemoglu et al., 2003; Hartley \& Hitti, 2005; Lindroos et al., 2018; Morley et al., 1989), we found a large effect of GA. In premature newborns, a low Apgar score may indicate intrinsic physiological immaturity and inadequate capacity for response rather than abnormal physiological functions (Iliodromiti et al., 2014). Preterm twins have the same prognosis as preterm singletons (Morley et al., 1989). The effect of GA was stronger than birth weight. We found effects of birth weight on Apgar scores in firstborn twins, but not in the second born.

Our findings on birth order agree with previous findings. The first twin is in better clinical condition (Franchi-Pinto et al., 1999; Haest et al., 2005; Herbst \& Kallen, 2008; Kwon et al., 2011; Morley et al., 1989; Thorngren-Jerneck \& Herbst, 2001; Usta et al., 2002; Wen et al., 2004). The second twin is at greater risk of lower scores, which can be due to longer delivery time, risk of hypoxia, nondefinable fetal presentation before birth to decide the better tactics or complications during delivery. The proportion of low Apgar score in first- and second born reported by Franchi-Pinto et al. (1999) corresponds with our findings. 
Fetal presentation at birth showed opposite effects in first- and second-born twins and should be further investigated, together with mode of delivery and in application of horizontal/nonhorizontal classification of presentation. The positive effect of noncephalic presentation in first-born twins in our study is in contrast with other studies (Hartley \& Hitti, 2005) and could be associated with tactics of delivery that can be planned in comparison with delivery of the second born. Delivery practice in the case of noncephalic presentation of the first born can increase the probability of high Apgar scores in the newborns. The previous studies have shown that the effect of fetal presentation on Apgar scores in the second born is associated with fetal presentation and mode of delivery of the first born (Bjelic-Radisic et al., 2007; Caukwell \& Murphy, 2002; Lindroos et al., 2018; Schmitz et al., 2008; Usta et al., 2002; Vogel et al., 2014). Cephalic presentation of the second twin is associated with higher Apgar scores in our study, in line with previous studies.

Physicians have gained a clear understanding of how to deliver twins with regard to their presentation and gestation. Some retrospective analyses and meta-analyses reported that the prognosis of twins was not different according to delivery mode (Hogle et al., 2003; Sibony et al., 2006; Usta et al., 2002), but population-based studies reported that the mortality rate or complications in second twins were higher in vaginal deliveries (Herbst \& Kallen, 2008; Jhaveri \& Nadkarni, 2016; Kwon et al., 2011; Rossi et al., 2011; Schmitz et al., 2008; Smith et al., 2002; Usta et al., 2005; Wen et al., 2004; Wenckus et al., 2014). We found better outcomes, in terms of Apgar score, for vaginal delivery in first-born twins and intervention delivery in the second born. We did not confirm that cesarean section is associated with low Apgar in second-born twins as shown by Wen et al. (2004). The mode of twin delivery should be considered on the basis of information on fetal presentation of both twins: the mode of delivery of the second born should take in account the mode of delivery of the first born.

To our knowledge, ours was the first large study evaluating genetic influences on 1- and 5-min Apgar scores. In contrast to a smaller twin study done without precise zygosity definition (Franchi-Pinto et al., 1999), we did not find evidence for genetic influences on Apgar scores. The slightly higher correlations in MZ twins in our study partly correspond with the intraclass correlation coefficients reported by Riese (1990) for 1-min Apgar scores in a small sample of MZ and DZ twins. We did observe large influences of nongenetic factors shared by twins from the same pairs. We acknowledge that a shared environmental component could reflect to some extent a shared measurement bias (e.g., if both twins are rated at the same time by one nurse). Apgar scores represent routine clinical practice, but some of the variability could reflect heterogeneity in clinical scoring practices as opposed to true differences in biomedical outcomes (Siddiqui et al., 2017). Also, the genotype of the mother in part creates the prenatal environment of both twins and thus is part of the 'shared environment'.

Our data do not reflect the whole population as it does not include cases with infant death. If the individual components of Apgar score (skin color or appearance, pulse rate, reflex, activity and respiratory effort) were available for analysis, it is possible that the contribution of shared and nonshared environment and genetic influence would differ across components. Twin-specific in utero environment and epigenetic factors are also of interest for future studies to examine the sources of unique environment. For understanding the variance of shared and nonshared perinatal environment, further analysis of mother's health status and early medical support is needed.

\section{Conclusions}

We have found that for both $\mathrm{MZ}$ and DZ pairs, second-born twins have lower Apgar scores in comparison with first-born twins. There are different effects of pre and perinatal characteristics on 1- and 5-min Apgar score in first- and second-born twins. Based on twin analyses, a genetic component was not significant for Apgar scores. For 1- and 5-min Apgar score, about half of the variation was explained by shared and half by nonshared environmental factors. It is possible that some of the shared environment is due to the same rate scoring both twins. The most important factors for Apgar scores are GA, birth weight, birth order, zygosity, fetal presentation and mode of delivery.

Supplementary material. To view supplementary material for this article, please visit https://doi.org/10.1017/thg.2019.24.

Acknowledgments. The authors would like to acknowledge the Netherlands Organization for Scientific Research (NWO) and the Netherlands Organization for Health Research and Development (ZonMW) grants: Twin family database for behavior genomics studies (NWO 480-04-004); Twin research focusing on behavior (NWO 400-05-717); Genotype/phenotype database for behavior genetic and genetic epidemiological studies (ZonMw Middelgroot 911-09-032); 'Why some children thrive' (OCW Gravity program NWO 024.001.003); Netherlands Twin Registry Repository: researching the interplay between genome and environment (NWO-Groot 480-15-001/674); Spinozapremie (NWO 56-464-14192) and KNAW Academy Professor Award (PAH/6635) to DIB ; Amsterdam Public Health (APH) and Amsterdam Reproduction \& Development (AR\&D).

Author contributions. VVO performed the analysis and wrote the manuscript; CVD designed the statistical analysis, performed the analysis and interpreted data; $\mathrm{CvB}$ prepared data set for analysis and commented on the results; JVD and EZ commented on results; DIB supervised the project, designed the manuscript and interpreted the data. All the authors interpreted the results, contributed to writing the manuscript and gave their consensus for submission.

Conflict of interest. The authors have no conflict of interest to declare.

\section{References}

Algeri, P., Callegari, C., Mastrolia, S. A., Brienza, L., Vaglio Tessitore, I., Paterlini, G., et al. (2018). What is the effect of intertwin delivery interval on the outcome of the second twin delivered vaginally? Journal of MaternalFetal \& Neonatal Medicine, 21, 1-7. doi: 10.1080/14767058.2018.1481036.

Apgar, V. (1953). A proposal for a new method of evaluation of the newborn infant. Current Researches in Anesthesia and Analgesia, 32, 260-267.

Apgar, V. (1966). The newborn (Apgar) scoring system. Reflections and advice. Pediatric Clinics of North America, 13, 645-650.

Barrett, J. F. R. (2013). A randomized trial of planned cesarean or vaginal delivery for twin pregnancy. New England Journal of Medicine, 369, $2364-$ 2364.

Bjelic-Radisic, V., Pristauz, G., Haas, J., Giuliani, A., Tamussino, K., Bader, A., ... Schlembach, D. (2007). Neonatal outcome of second twins depending on presentation and mode of delivery. Twin Research and Human Genetics, 10, 521-527.

Bodnar, L. M., Pugh, S. J., Abrams, B., Himes, K. P., \& Hutcheon, J. A. (2014). Gestational weight gain in twin pregnancies and maternal and child health: A systematic review. Journal of Perinatology, 34, 252-263.

Boomsma, D., Busjahn, A., \& Peltonen, L. (2002). Classical twin studies and beyond. Nature Reviews Genetics, 3, 872-882.

Camilleri, A. P. (1981). The obstetric significance of short stature. European Journal of Obstetrics \& Gynecology and Reproductive Biology, 12, 347-356.

Caserta, D., Bordi, G., Stegagno, M., Filippini, F., Podagrosi, M., Roselli, D., ... Moscarini, M. (2014). Maternal and perinatal outcomes in spontaneous versus assisted conception twin pregnancies. European Journal of Obstetrics \& Gynecology and Reproductive Biology, 174, 64-69. 
Casey, B. M., McIntire, D. D., \& Leveno, K. J. (2001). The continuing value of the Apgar score for the assessment of newborn infants. New England Journal of Medicine, 344, 467-471.

Caukwell, S., \& Murphy, D. J. (2002). The effect of mode of delivery and gestational age on neonatal outcome of the non-cephalic- presenting second twin. American Journal of Obstetrics and Gynecology, 187, 1356-1361.

Chen, M., McNiff, C., Madan, J., Goodman, E., Davis, J. M., \& Dammann, O. (2010). Maternal obesity and neonatal Apgar scores. Journal of MaternalFetal and Neonatal Medicine, 23, 89-95.

Chen, X. K., Wen, S. W., Fleming, N., Demissie, K., Rhoads, G. G., \& Walker, M. (2007). Teenage pregnancy and adverse birth outcomes: A large population based retrospective cohort study. International Journal of Epidemiology, $36,368-373$.

Committee on Obstetric Practice American Academy of Pediatrics Committee on Fetus and Newborn. (2015). Committee opinion No. 644: The Apgar score. Obstetrics \& Gynecology, 126, e52-55.

Daniel, Y., Ochshorn, Y., Fait, G., Geva, E., Bar-Am, A., \& Lessing, J. B. (2000). Analysis of 104 twin pregnancies conceived with assisted reproductive technologies and 193 spontaneously conceived twin pregnancies. Fertility and Sterility, 74, 683-689.

Dolgun, Z. N., Inan, C., Altintas, A. S., Okten, S. B., \& Sayin, N. C. (2016). Preterm birth in twin pregnancies: Clinical outcomes and predictive parameters. Pakistan Journal of Medical Sciences, 32, 922-926.

Drage, J. S., Kennedy, C., \& Schwarz, B. K. (1964). The Apgar score as an index of neonatal mortality. A report from the Collaborative Study of Cerebral Palsy. Obstetrics \& Gynecology, 24, 222-230.

Dube, J., Dodds, L., \& Armson, B. A. (2002). Does chorionicity or zygosity predict adverse perinatal outcomes in twins? American Journal of Obstetrics and Gynecology, 186, 579-583.

Erdemoglu, E., Mungan, T., Tapisiz, O. L., Ustunyurt, E., \& Caglar, E. (2003). Effect of inter-twin delivery time on Apgar scores of the second twin. Australian and New Zealand Journal of Obstetrics and Gynaecology, 43, 203-206.

Falconer, D. (1993). Quantitative genetics in Edinburgh: 1947-1980. Genetics, 133, 137-142.

Fan, C., Sun, Y., Yang, J., Ye, J., \& Wang, S. (2013). Maternal and neonatal outcomes in dichorionic twin pregnancies following IVF treatment: A hospital-based comparative study. International Journal of Clinical and Experimental Pathology, 6, 2199-2207.

Fox, N. S., Silverstein, M., Bender, S., Klauser, C. K., Saltzman, D. H., \& Rebarber, A. (2010). Active second-stage management in twin pregnancies undergoing planned vaginal delivery in a U.S. population. Obstetrics \& Gynecology, 115, 229-233.

Franchi-Pinto, C., Dal Colletto, G. M. D., Krieger, H., \& Beiguelman, B. (1999). Genetic effect on Apgar score. Genetics and Molecular Biology, 22, 13-16.

Gilman, S. E., Gardener, H., \& Buka, S. L. (2008). Maternal smoking during pregnancy and children's cognitive and physical development: A causal risk factor? American Journal of Epidemiology, 168, 522-531.

Groen-Blokhuis, M. M., Middeldorp, C. M., van Beijsterveldt, C. E., \& Boomsma, D. I. (2011). Evidence for a causal association of low birth weight and attention problems. Journal of the American Academy of Child and Adolescent Psychiatry, 50, 1247-1254.

Grunebaum, A., McCullough, L. B., Sapra, K. J., Brent, R. L., Levene, M. I., Arabin, B., \& Chervenak, F. A. (2013). Apgar score of 0 at 5 minutes and neonatal seizures or serious neurologic dysfunction in relation to birth setting. American Journal of Obstetrics and Gynecology, 209, 323 e321-326.

Haest, K. M. J., Roumen, F. J. M. E., \& Nijhuis, J. G. (2005). Neonatal and maternal outcomes in twin gestations 32 weeks according to the planned mode of delivery. European Journal of Obstetrics \& Gynecology and Reproductive Biology, 123, 17-21.

Harrington, D. J., Redman, C. W., Moulden, M., \& Greenwood, C. E. (2007). The long-term outcome in surviving infants with Apgar zero at 10 minutes: A systematic review of the literature and hospital-based cohort. American Journal of Obstetrics and Gynecology, 196, 463 e461-465.

Hartley, R. S., \& Hitti, J. (2005). Birth order and delivery interval: Analysis of twin pair perinatal outcomes. Journal of Maternal-Fetal and Neonatal Medicine, 17, 375-380.

Hegyi, T., Carbone, T., Anwar, M., Ostfeld, B., Hiatt, M., Koons, A., ... Paneth, N. (1998). The Apgar score and its components in the preterm infant. Pediatrics, 101(1 Pt 1), 77-81.
Hemminki, E., Malin, M., \& Rahkonen, O. (1990). Mother's social class and perinatal problems in a low-problem area. International Journal of Epidemiology, 19, 983-990.

Herbst, A., \& Kallen, K. (2008). Influence of mode of delivery on neonatal mortality in the second twin, at and before term. BJOG, 115, 1512-1517.

Hjorto, S., Nickelsen, C., Petersen, J., \& Secher, N. J. (2014). The effect of chorionicity and twin-to-twin delivery time interval on short-term outcome of the second twin. Journal of Maternal-Fetal and Neonatal Medicine, 27, 42-47.

Hogle, K. L., Hutton, E. K., McBrien, K. A., Barrett, J. F., \& Hannah, M. E. (2003). Cesarean delivery for twins: A systematic review and meta-analysis. American Journal of Obstetrics and Gynecology, 188, 220-227.

Iliodromiti, S., Mackay, D. F., Smith, G. C., Pell, J. P., \& Nelson, S. M. (2014). Apgar score and the risk of cause-specific infant mortality: A populationbased cohort study. Lancet, 384, 1749-1755.

Jahromi, B. N., \& Husseini, Z. (2008). Pregnancy outcome at maternal age 40 and older. Taiwanese Journal of Obstetrics and Gynecology, 47, 318-321.

Jhaveri, R. R., \& Nadkarni, T. K. (2016). Perinatal outcome of second twin with respect to mode of delivery: An observational study. Journal of Clinical and Diagnostic Research, 10, qc26-qc28.

Kalland, M., Sinkkonen, J., Gissler, M., Merilainen, J., \& Siimes, M. A. (2006). Maternal smoking behavior, background and neonatal health in Finnish children subsequently placed in foster care. Child Abuse \& Neglect, 30, 1037-1047.

Kallen, K. (2001). The impact of maternal smoking during pregnancy on delivery outcome. European Journal of Public Health, 11, 329-333.

Kattwinkel, J., Perlman, J. M., Aziz, K., Colby, C., Fairchild, K., Gallagher, J., Zaichkin, J. (2010). Part 15: Neonatal Resuscitation 2010 American Heart Association Guidelines for Cardiopulmonary Resuscitation and Emergency Cardiovascular Care. Circulation, 122, S909-S919.

Khandwala, Y. S., Baker, V. L., Shaw, G. M., Stevenson, D. K., Lu, Y., \& Eisenberg, M. L. (2018). Association of paternal age with perinatal outcomes between 2007 and 2016 in the United States: Population based cohort study. $B M J, 363, \mathrm{k} 4372$.

Kiran, T. S. U., Hemmadi, S., Bethel, J., \& Evans, J. (2005). Outcome of pregnancy in a woman with an increased body mass index. BJOG - An International Journal of Obstetrics and Gynaecology, 112, 768-772.

Koudstaal, J., Bruinse, H. W., Helmerhorst, F. M., Vermeiden, J. P., Willemsen, W. N., \& Visser, G. H. (2000). Obstetric outcome of twin pregnancies after in-vitro fertilization: A matched control study in four Dutch university hospitals. Human Reproduction, 15, 935-940.

Krebs, L., \& Langhoff-Roos, J. (1999). Breech delivery at term in Denmark, 1982-92: A population-based case-control study. Paediatric and Perinatal Epidemiology, 13, 431-441.

Krebs, L., Langhoff-Roos, J., \& Thorngren-Jerneck, K. (2001). Long-term outcome in term breech infants with low Apgar score - A population-based follow-up. European Journal of Obstetrics \& Gynecology and Reproductive Biology, 100, 5-8.

Kwon, J. Y., Yoon, W. S., Lee, G. S., Kim, S. J., Shin, J. C., \& Park, I. Y. (2011). Umbilical arterial blood gas and perinatal outcome in the second twin according to the planned mode of delivery. International Journal of Medical Sciences, 8, 643-648.

Ladehoff, P., Pedersen, G. T., \& Sorensen, T. (1986). Apgar scores in low birth weight infants delivered vaginally and by cesarean section. Acta Obstetricia et Gynecologica Scandinavica, 65, 3-5.

Li, F., Wu, T., Lei, X., Zhang, H., Mao, M., \& Zhang, J. (2013). The Apgar score and infant mortality. PLoS One, 8, e69072.

Lindroos, L., Elfvin, A., Ladfors, L., \& Wennerholm, U. B. (2018). The effect of twin-to-twin delivery time intervals on neonatal outcome for second twins. BMC Pregnancy Childbirth, 18, 36.

Machado, M., Lima Teixeira, E., Ferreira, L. M., Rodrigues, F., Henriques, R., \& Afonso, E. (2017). Perinatal outcome in relation to chorionicity in twin pregnancy. Acta Médica Portuguesa, 30, 12-16.

Marbury, M. C., Linn, S., Monson, R. R., Wegman, D. H., Schoenbaum, S. C., Stubblefield, P. G., ... Ryan, K. J. (1984). Work and pregnancy. Journal of Occupational Medicine, 26, 415-421.

Milsom, I., Ladfors, L., Thiringer, K., Niklasson, A., Odeback, A., \& Thornberg, E. (2002). Influence of maternal, obstetric and fetal risk factors on the prevalence of birth asphyxia at term in a Swedish urban population. Acta Obstetricia et Gynecologica Scandinavica, 81, 909-917. 
Moise, J., Laor, A., Armon, Y., Gur, I., \& Gale, R. (1998). The outcome of twin pregnancies after IVF. Human Reproduction, 13, 1702-1705.

Morley, R., Cole, T. J., Powell, R., \& Lucas, A. (1989). Growth and development in premature twins. Archives of Disease in Childhood, 64, 1042-1045.

Muthen, L. K., \& Muthen, B. O. (2007). Mplus user's guide (6th ed.). Los Angeles, CA:Muthen and Muthen.

Nelson, K. B., \& Ellenberg, J. H. (1981). Apgar scores as predictors of chronic neurologic disability. Pediatrics, 68, 36-44.

O'Donnell, C. P., Kamlin, C. O., Davis, P. G., Carlin, J. B., \& Morley, C. J. (2006). Interobserver variability of the 5-minute Apgar score. Journal of Pediatrics, 149, 486-489.

Ochsenkuhn, R., Strowitzki, T., Gurtner, M., Strauss, A., Schulze, A., Hepp, H., ... Hillemanns, P. (2003). Pregnancy complications, obstetric risks, and neonatal outcome in singleton and twin pregnancies after GIFT and IVF. Archives of Gynecology and Obstetrics, 268, 256-261.

Odd, D. E., Doyle, P., Gunnell, D., Lewis, G., Whitelaw, A., \& Rasmussen, F. (2008). Risk of low Apgar score and socioeconomic position: a study of Swedish male births. Acta Paediatrica, 97, 1275-1280.

Odintsova, V. V., Willemsen, G., Dolan, C. V., Hottenga, J. J., Martin, N. G., Slagboom, P. E., ... Boomsma, D. I. (2018). Establishing a twin register: An invaluable resource for (behavior) genetic, epidemiological, biomarker, and 'omics' studies. Twin Research and Human Genetics, 21, 239-252.

Pourali, L., Ayati, S., Jelodar, S., Zarifian, A., \& Andalibi, M. S. S. (2016). Obstetrics and perinatal outcomes of dichorionic twin pregnancy following ART compared with spontaneous pregnancy. International Journal of Reproductive Biomedicine, 14, 317-322.

Ramoglu, M. G., Kavuncuoglu, S., Ozbek, S., \& Aldemir, E. (2014). Perinatal and somatic growth properties of preterm babies born from spontaneous and in vitro fertilization multiple pregnancies. Turkish Archives of Pediatrics, 49, $17-24$.

Rayburn, W. F., Lavin, J. P., Jr., Miodovnik, M., \& Varner, M. W. (1984). Multiple gestation: time interval between delivery of the first and second twins. Obstetrics \& Gynecology, 63, 502-506.

Riese, M. L. (1990). Genetic influences on neonatal temperament. Acta Geneticae Medicae et Gemellologiae, 39, 207-213.

Rietveld, M. J., van Der Valk, J. C., Bongers, I. L., Stroet, T. M., Slagboom, P. E., \& Boomsma, D. I. (2000). Zygosity diagnosis in young twins by parental report. Twin Research, 3, 134-141.

Rode, L., Nilas, L., Wojdemann, K., \& Tabor, A. (2005). Obesity-related complications in Danish single cephalic term pregnancies. Obstetrics \& Gynecology, 105, 537-542.

Rossi, A. C., Mullin, P. M., \& Chmait, R. H. (2011). Neonatal outcomes of twins according to birth order, presentation and mode of delivery: A systematic review and meta-analysis. BJOG, 118, 523-532.

Rudiger, M., Konstantelos, D., \& Consortium, T.-A. (2015). Apgar score and risk of cause-specific infant mortality. Lancet, 385, 505-506.

Schmitz, T., Carnavalet Cde, C., Azria, E., Lopez, E., Cabrol, D., \& Goffinet, F. (2008). Neonatal outcomes of twin pregnancy according to the planned mode of delivery. Obstetrics \& Gynecology, 111, 695-703.

Schneuber, S., Magnet, E., Haas, J., Giuliani, A., Freidl, T., Lang, U., ... Bjelic-Radisic, V. (2011). Twin-to-twin delivery time: Neonatal outcome of the second twin. Twin Research and Human Genetics, 14, 573-579.

Sentilhes, L., Goffinet, F., Talbot, A., Diguet, A., Verspyck, E., Cabrol, D., .. Marpeau, L. (2007). Attempted vaginal versus planned cesarean delivery in 195 breech first twin pregnancies. Acta Obstetricia et Gynecologica Scandinavica, 86, 55-60.

Sibony, O., Touitou, S., Luton, D., Oury, J. F., \& Blot, P. (2006). Modes of delivery of first and second twins as a function of their presentation. Study of 614 consecutive patients from 1992 to 2000. European Journal of Obstetrics \& Gynecology and Reproductive Biology, 126, 180-185.

Siddiqui, A., Cuttini, M., Wood, R., Velebil, P., Delnord, M., Zile, I., ... Comm, E.-P. S. (2017). Can the Apgar score be used for international comparisons of newborn health? Paediatric and Perinatal Epidemiology, $31,338-345$.
Smith, G. C., Pell, J. P., \& Dobbie, R. (2002). Birth order, gestational age, and risk of delivery related perinatal death in twins: Retrospective cohort study. BMJ, 325, 1004.

Stein, W., Misselwitz, B., \& Schmidt, S. (2008). Twin-to-twin delivery time interval: Influencing factors and effect on short-term outcome of the second twin. Acta Obstetricia et Gynecologica Scandinavica, 87, 346-353.

Stevenson, D. K., Verter, J., Fanaroff, A. A., Oh, W., Ehrenkranz, R. A., Shankaran, S., ... Papile, L. (2000). Sex differences in outcomes of very low birthweight infants: The newborn male disadvantage. Archives of Disease in Childhood, 83, F182-F185.

Straube, S., Voigt, M., Jorch, G., Hallier, E., Briese, V., \& Borchardt, U. (2010). Investigation of the association of Apgar score with maternal socioeconomic and biological factors: An analysis of German perinatal statistics. Archives of Gynecology and Obstetrics, 282, 135-141.

Svenvik, M., Brudin, L., \& Blomberg, M. (2015). Preterm birth: A prominent risk factor for low Apgarscores. Biomed Research International, 2015, Article ID 978079, doi: 10.1155/2015/978079

Tan, H., Wen, S. W., Walker, M., \& Demissie, K. (2004). Missing paternal demographics: A novel indicator for identifying high risk population of adverse pregnancy outcomes. BMC Pregnancy Childbirth, 4, 21.

Thorngren-Jerneck, K., \& Herbst, A. (2001). Low 5-minute Apgar score: A population-based register study of 1 million term births. Obstetrics \& Gynecology, 98, 65-70.

Usta, I. M., Nassar, A. H., Awwad, J. T., Nakad, T. I., Khalil, A. M., \& Karam, K. S. (2002). Comparison of the perinatal morbidity and mortality of the presenting twin and its co-twin. Journal of Perinatology, 22, 391-396.

Usta, I. M., Rechdan, J. B., Khalil, A. M., \& Nassar, A. H. (2005). Mode of delivery for vertex-nonvertex twin gestations. International Journal of Gynecology and Obstetrics, 88, 9-14.

van Beijsterveldt, C. E., Groen-Blokhuis, M., Hottenga, J. J., Franic, S., Hudziak, J. J., Lamb, D., .. Boomsma, D. I. (2013). The Young Netherlands Twin Register (YNTR): Longitudinal twin and family studies in over 70,000 children. Twin Research and Human Genetics, 16, 252-267.

van Beijsterveldt, C. E., Overbeek, L. I., Rozendaal, L., McMaster, M. T., Glasner, T. J., Bartels, M., ... Boomsma, D. I. (2016). Chorionicity and heritability estimates from twin studies: The prenatal environment of twins and their resemblance across a large number of traits. Behavior Genetics, 46, 304-314.

van der Ven, A. J., Schaaf, J. M., van Os, M. A., de Groot, C. J., Haak, M. C., Pajkrt, E., .. Mol, B. W. (2014). Comparison of perinatal outcome of preterm births starting in primary care versus secondary care in Netherlands: A retrospective analysis of nationwide collected data. Obstetrics and Gynecology International, 2014, Article ID 423575, doi: 10.1155/2014/423575

van Dijk, B. A., Boomsma, D. I., \& de Man, A. J. (1996). Blood group chimerism in human multiple births is not rare. American Journal of Medical Genetics, 61, 264-268.

Vogel, J. P., Holloway, E., Cuesta, C., Carroli, G., Souza, J. P., \& Barrett, J. (2014). Outcomes of non-vertex second twins, following vertex vaginal delivery of first twin: A secondary analysis of the WHO Global Survey on maternal and perinatal health. BMC Pregnancy Childbirth, 14, 55.

Vulic, M., Lalic, L., Vulic, L., Roje, D., Benzon, Z., \& Mestrovic, Z. (2017). A retrospective study of discordant twin growth in dichorionic twin pregnancies and risk of preterm delivery at Split University Hospital Centre: Threeyear experience. Acta Clinica Croatica, 56, 640-644.

Wen, S. W., Fung Kee Fung, K., Oppenheimer, L., Demissie, K., Yang, Q., \& Walker, M. (2004). Neonatal morbidity in second twin according to gestational age at birth and mode of delivery. American Journal of Obstetrics and Gynecology, 191, 773-777.

Wenckus, D. J., Gao, W., Kominiarek, M. A., \& Wilkins, I. (2014). The effects of labor and delivery on maternal and neonatal outcomes in term twins: A retrospective cohort study. BJOG, 121, 1137-1144.

World Health Organization (WHO). (2012). Guidelines on Basic Newborn Resuscitation. Geneva, Switzerland: World Health Organization.

Zhao, D., Zou, L., Lei, X., \& Zhang, Y. (2017). Gender differences in infant mortality and neonatal morbidity in mixed-gender twins. Scientific Reports, $7(1), 8736$ 